\title{
Astroparticle Physics with ARGO-YBJ experiment
}

\author{
Giovanni Marsella ${ }^{1}$ (on behalf of ARGO-YBJ coll.) \\ Università del Salento and INFN \\ Address, Country \\ E-mail: giovanni.marsellalle.infn.it
}

The ARGO-YBJ experiment, installed at the Yangbajing Cosmic Ray Laboratory (Tibet, China), at $4300 \mathrm{~m}$ a.s.l., is a detector $100 \times 110 \mathrm{~m}^{2}$ large, made by a layer of Resistive Plate Counters (RPCs) consisting of a central carpet with almost full coverage extending over an area of about $5.500 \mathrm{~m}^{2}$, surrounded by a guard ring with partial coverage. The high space-time granularity, the full-coverage technique and the high altitude location make this detector a unique device for a detailed study of the atmospheric shower characteristics with an energy threshold of a few hundred GeV. These properties in addition to the large field of view and the high duty cycle enable the ARGO-YBJ experiment to monitor the sky in a continuous way. Results have been reached in a wide variety of fields ranging from Gamma Astronomy, to Solar Physics, from Cosmic Rays composition to hadronic interactions and proton-antiproton ratio. A summary of all these results will be presented and reviewed.

35th International Conference of High Energy Physics (ICHEP2010)

Paris,France

July 22-28, 2010

\footnotetext{
$1 \quad$ Speaker
} 


\section{Introduction}

The ARGO-YBJ (Astrophysical Radiation with Ground-based Observatory at YangBaJing) experiment is operating at the Yangbajing High Altitude Cosmic Ray Laboratory (4300 m a.s.1.), situated $90 \mathrm{~km}$ in the North of Lhasa (Tibet, P.R.China), as an Italian-Chinese collaboration project. It is operating since June 2006 with the aim of studying cosmic rays, mainly cosmic radiation, at an energy threshold of a few hundreds $\mathrm{GeV}$, by detecting small size air showers at high altitude with wide aperture and high duty cycle. It is made of a single layer of Resistive Plate Counters (RPCs)[1] fully covering an area $74 \times 78 \mathrm{~m}^{2}$. In order to improve the apparatus performance in the detection of showers using the central carpet, especially with better core location resolution, the actual area is enlarged by partially surrounding the central detector with a guard ring of RPCs, up to $100 \times 110 \mathrm{~m}^{2}$. The detector is configured in modules of 12 RPCs, each RPC of dimensions $280 \times 125 \mathrm{~cm}^{2}$. This group of RPCs, called 'Cluster', is the basic detection and Data Acquisition unit in a logical subdivision of the apparatus. The percentage of active area in the central detector, made of 130 Clusters, is $92 \%$. Each RPC is divided into 10 digital pick-up pads $56 \times 62 \mathrm{~cm}^{2}$ (subdivided in 8 strips) with a time resolution $\leq$ 2 ns. For each event the location and timing of every hit pad is recorded, allowing the lateral distribution and arrival direction reconstruction. The trigger is set to a multiplicity $\geq 20$ hits in $420 \mathrm{~ns}$, collecting events at a rate of $\sim 3.6 \mathrm{kHz}$. The detector can also be operated in a 'Scalar Mode', where for each cluster the signal coming from the 120 pads within $150 \mathrm{~ns}$ are added up. The full detector counts are measured every $0.5 \mathrm{~s}$, without any information on both the space distribution and arrival direction of the detected particles. A detailed status report of the experiment can be found in [2].

\section{The moon shadow}

Cosmic Rays are hampered by the moon, so a deficit in the cosmic ray flux is observed in its direction. The moon shadow is the strongest signal observed in the sky by ARGO-YBJ detector. The measurement of the size of the deficit allows defining the angular resolution of the apparatus, while its position indicates the pointing accuracy. The presence of the Earth magnetic field deviates eastward the charged particles travelling from the moon toward the Earth with an approximate dependence from the energy expressed by $\Delta \theta \sim \mathrm{Z}$ x $1.6^{\circ} / \mathrm{E}(\mathrm{TeV})$. The observation of the westward displacement of the moon shadow in function of multiplicity allows energy calibration of the detector. The technique is used also to estimate the proton antiproton ratio in cosmic rays. Actually ARGO-YBJ has collected data continuously with sensitivity to the moon shadow of about $10 \sigma /$ month. At the end of 2009, $3200 \mathrm{~h}$ of data on source were integrated, reaching a sensitivity of 55 sigma selecting events with Number of fired strips $>100$ and the zenith angle $\theta \leq 50^{\circ}$. In fig. 1 the angular resolution and energy relation to the number of strips of the event are shown. At energy $<10 \mathrm{TeV}$, the uncertainty in energy estimation is less than $18 \%$, an impressive result for an EAS detector. The following limits on proton-antiproton ratio have been obtained at 90\% C.L.: between 60 and100 fired strips (median E 2 TeV) an upper limit of 4.2\%, between 100 and 50000 (median $\mathrm{E} \sim 5 \mathrm{TeV}$ ) an upper limit of 7.4\%. 

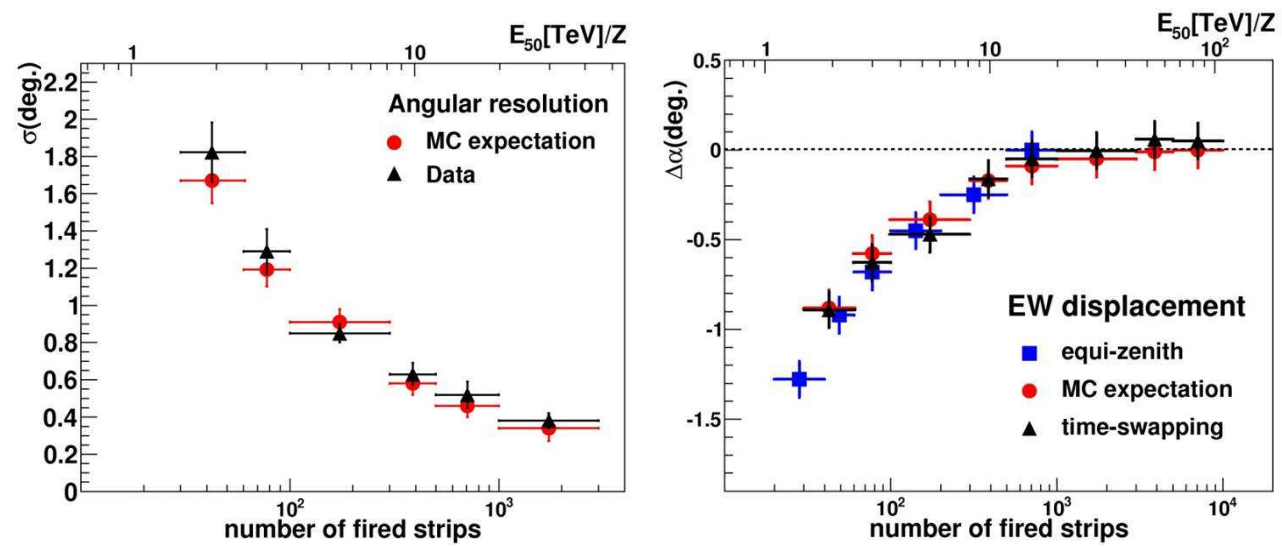

Fig. 1: Angular resolution and EW displacement in function of the number of fired strips(and corresponding energy evaluation)

\section{Cosmic rays}

Regards to cosmic ray topics, the first results of the ARGO-YBJ experiment are the measurement of proton-air cross section, the measurement of the primary CR light component and the proton sky map anisotropies.

The proton-air cross section measurement analysis is based on the shower flux attenuation for different zenith angles and exploits the detector accuracy in reconstructing the shower properties. For fixed primary energy and shower age, such attenuation is expressed by the absorption length, $\Lambda$, connected to the primary interaction length in the atmosphere by the relation: $\Lambda=\mathrm{k} \cdot \lambda_{\mathrm{INT}}$ where $\mathrm{k}$ depends on the shower development in the atmosphere, on its fluctuations and on the detector response. The actual value of $\mathrm{k}$ has been evaluated by Monte Carlo simulations, and it might in principle depend on the features of the adopted hadronic interaction model. For primary protons, the interaction length is related to the p-air interaction cross-section by: $\sigma_{\mathrm{p}-\mathrm{AIR}}[\mathrm{mb}]=2.41 \times 10^{4} / \lambda_{\text {INT }}$.

The ARGO-YBJ detector location and features, which ensure the capability of reconstructing the detected showers in a very detailed way, has been exploited (see [3] for the analysis details). All the possible systematic sources have been taken into account and evaluated on the basis of a full Monte Carlo simulation. Finally, Glauber theory has been used to infer the total proton-proton cross section from the measured proton-air cross section, in an energy region not yet explored by accelerator experiments. It permit better investigating the behaviour of the p-p total cross section where it starts to significantly increase with energy. In particular, our result is compatible with the asymptotic squared logarithmic increase of total hadronic cross section as obtained in ref[4] and ref[5] from a global analysis of accelerator data.

A first measurement of the differential energy spectrum of the primary CR light component $(p+H e)$ has been performed by ARGO-YBJ applying a Bayesian unfolding approach to the strip multiplicity spectrum. Showers with zenith angle $<30^{\circ}$ and with reconstructed core position inside a fiducial area $50 \times 50 \mathrm{~m}^{2}$ large by applying a selection criterion based on the fired strip density. In the investigated energy range, the contamination of nuclei heavier than Helium is found not exceeding a few percent. Anyway, the present analysis does not allow the 
determination of the individual proton and Helium contribution to the measured flux. The ARGO-YBJ measurements agree remarkably well with the values obtained by adding up the proton and helium fluxes measured by the CREAM experiment in the same energy range, concerning both the total intensities and the spectrum [6]. The value of the spectral index of the power-law fit representing the ARGO-YBJ data is $-2.61 \pm 0.04$, which should be compared to $\gamma_{\mathrm{p}}$ $=-2.66 \pm 0.02$ and $\gamma_{\mathrm{He}}=-2.58 \pm 0.02$ obtained by the CREAM experiment. It is the first time that direct and ground based measurements overlap for a wide energy range making possible the cross-calibration of different experimental techniques.

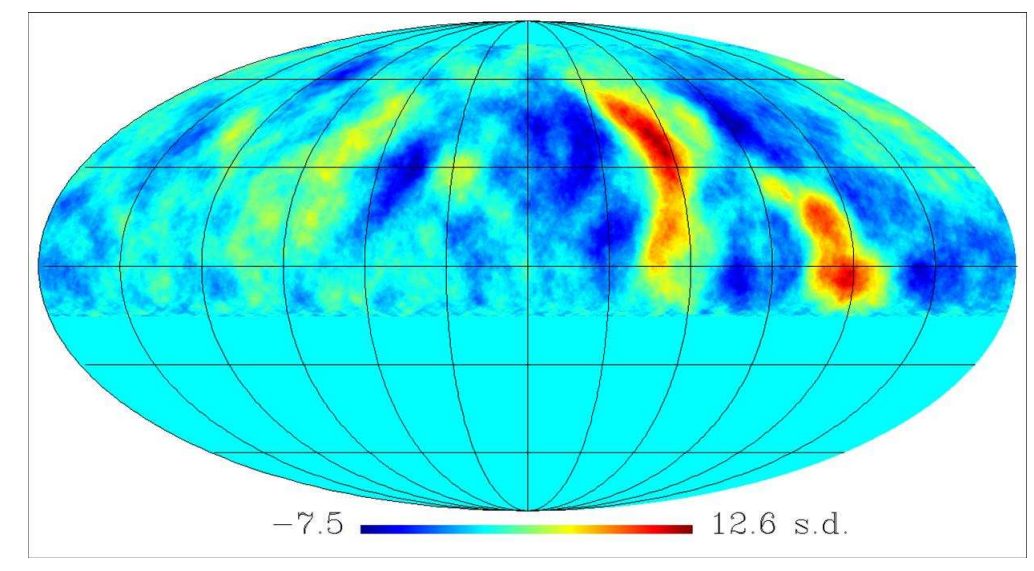

Fig. 2: ARGO-YBJ medium scale anisotropy of Cosmic Rays at $\sim 2 \mathrm{TeV}$.

Galactic Cosmic Rays at $\mathrm{TeV}$ energies are expected to appear highly isotropic, since during their propagation from the sources to the Earth they undergo complex processes, such as the deflection by the large scale Galactic magnetic field and the interaction with background photons and interstellar medium. The study of the galactic CR anisotropy is a useful tool in probing the magnetic field structure in our interstellar neighbourhood as well as the source distribution. Large and medium scale anisotropies have been reported by several experiments. In this work the ARGO-YBJ medium scale anisotropy observation is reported. The analysis has been performed in order to be insensitive to the CR large-scale anisotropy which is roughly one order of magnitude greater. The map in fig. 2 clearly shows two large hot spots in the region of the Galactic anticenter. The two excesses (>10 s.d., corresponding to a flux increase of $\sim 0.1 \%$ ), observed by ARGO-YBJ around the positions ra $\sim 120^{\circ}$, dec $\sim 40^{\circ}$ and $\mathrm{ra} \sim 60^{\circ}$, dec $\sim-5^{\circ}$, are in agreement with a similar detection reported by the Milagro Collaboration also if some little differences are evident and probably due to the fact that ARGO-YBJ can observe the southern regions of the sky with more efficiency, being located at a lower latitude than Milagro.

\section{Gamma astronomy}

Another important task of ARGO-YBJ detector is the Gamma Astronomy with an energy threshold of few hundreds of GeV. An all sky survey in 825 days of observation (jul 06. - oct. 2009) has evidenced 3 sources at a significance $>5 \sigma$. The analysis has been done using the direct integral method to evaluate the background and a multiplicity selection requiring more 
then 40 fired strips. No gamma/hadron selections have been applied. The three sources are Crab, Mrk421 and MGRO1908+06 which have been detected with the significances of $14.5 \sigma, 11.9 \sigma$ and $5.4 \sigma$ respectively. So the detector, without any particular gamma/hadron selection, has a sensitivity of about $50 \%$ of the Crab flux per year. A Crab Nebula differential spectrum has been determined: $\mathrm{dN} / \mathrm{dE}=(3.0 \pm 0.3) \cdot 10^{-11} \cdot \mathrm{E}^{-2.74 \pm 0.14} \mathrm{ph} \mathrm{cm}^{-2} \mathrm{~s}^{-1} \mathrm{TeV}^{-1}$.

Mrk421 has been monitored on a long time scale and several flares have been detected. In particular in 2008 its flare activity was particularly intense. Flares were observed in February and in June. A spectrum was calculated from data collected between day 40 and 180 in 2008: $\mathrm{dN} / \mathrm{dE}=(7.5 \pm 1.7)^{\prime} 10^{-11} \mathrm{E}^{-2.51 \pm 0.29} \mathrm{e}^{-\tau(\mathrm{E})} \mathrm{cm}^{-2} \mathrm{~s}^{-1} \mathrm{TeV}^{-1}$ taking into account the EBL absorption, while during the June flare period a multi wavelength analysis has been done finding fairly good agreement in light curves with the lower spectrum band, contributing to the model study of flare mechanisms. In particular, during the second flare in June 2008 (between 13 and 15) ARGO was the only $\mathrm{TeV}$ active detector, as in these days the Cerenkov were blinded by the Moon, demonstrating the importance of having detectors allowing a long term and continuous monitoring of the sky[7].

The observation of MGRO1908+06, discovered by MILAGRO and confirmed by Hess and Veritas, is particularly important in order to solve the puzzle of the discrepancy showed by the different techniques. The observed signal seems to confirm MILAGRO results. Also the absence of signal detected by ARGO-YBJ from other sources observed by MILAGRO must be analysed in order to correctly define relative sensibility of the detectors and the nature of the observed signals.

\section{Conclusions}

ARGO-YBJ detector is taking data steadily since 2006 at rate of $\sim 3.6 \mathrm{kHz}$ with a duty cycle close to $90 \%$. The Moon shadow is continuously monitored with a sensitivity of about 10 $\sigma$ per month, reaching, at the end of 2009, a sensitivity of $55 \sigma$, which allows a good detector calibration of angular resolution, pointing accuracy and energy determination. From the study of the shape of the moon shadow a first limit on proton/antiproton ratio in cosmic rays has been calculated. In cosmic ray physics the total proton-proton cross section has been measured. The result is obtained in an energy region not yet covered by accelerators and seems to be consistent with models expecting a $\ln ^{2}(\mathrm{~S})$ asymptotic behaviour. A first light component spectrum has been measured using a Bayesian unfolding method in an energy range between 5 and $250 \mathrm{TeV}$. The result is in quite good agreement with CREAM measurements. Medium scale anisotropy in cosmic rays has been observed with a significance of $10 \sigma$ at a mean energy of about $2 \mathrm{TeV}$. In Gamma Ray astronomy after 825 days of sky survey, without any gamma/hadron selection, 3 sources have been detected with significance greater than $5 \sigma$.

\section{References}

[1] G. Aielli et al., Nucl. Inst. and Meth in Phys. Res. A 562, p92, 2006

[2] G. Aielli et al., Astrop. Phys. 32, 47, 2009.

[3] G. Aielli et al., Phys. Rev. D 80, 092004, 2009. 
[4] C. Amsler et al. , Phys. Lett., B 667, 1, $2008 .$.

[5] M.M Block and F. Halzen, Phys. Rev. D 72, 036006, 2005.

[6] H. S. Ahn, et al., Astrophys. J. 715, $1400-14072010$.

[7] G.Aielli et al., Astrophysical Journal Letters 714, L208, 2010 